\title{
Neurovascular uncoupling: much ado about nothing
}

\section{Nikos K. Logothetis ${ }^{1,2 *}$}

1 Max Planck Institute for Biological Cybernetics, Tuebingen, Germany

2 Division of Imaging Science and Biomedical Engineering, University of Manchester, Manchester, UK

*Correspondence: nikos.logothetis@tuebingen.mpg.de
The vast majority of human fMRI studies measure Blood-Oxygen-Level-Dependent (BOLD) contrast, which reflects regional changes in cerebral blood flow (CBF), cerebral blood volume (CBV) and blood oxygenation; all three vascular responses reflect local increases in neural activity (Logothetis and Wandell, 2004). Understanding the exact mechanism (often referred to as neurovascular coupling) by means of which changes in neural activity alter hemodynamics is obviously of paramount importance for the meaningful interpretation of fMRI results. Not surprisingly, over the last decade an increasing number of researchers investigated the neurovascular coupling by combining fMRI with electroencephalography (EEG) or magnetoencephalography (MEG) in humans, e.g. (Dale and Halgren, 2001) as well as with intracortical recordings in animals (Logothetis et al., 2001; Goense and Logothetis, 2008; Logothetis, 2008; Rauch et al., 2008). This neurovascular coupling can also be studied with the optical imaging of intrinsic signals (OIS) (Bonhoeffer and Grinvald, 1996), an excellent invasive method of high spatiotemporal resolution that can measure changes in oxygenation and/or blood volume.

Recently, an OIS variant permitting simultaneous measurements of blood volume and oxygenation was used to image the visual cortex of behaving monkeys performing visual or auditory tasks (Sirotin and Das, 2009). Concurrent hemodynamic measurements were performed by rapidly switching between two wavelengths, $530 \mathrm{~nm}$ and $605 \mathrm{~nm}$, permitting the measurement of $\mathrm{CBV}$ and oxygenation, respectively. During the measurements the monkeys were required to periodically direct their gaze to a point source in order to receive a juice reward. The fixation point remained on continuously, but switched between two equiluminant colors to cue the animal to 'fixate' (green) or 'relax' (red). In one task, the fixation spot was followed by a brief visual stimulus presentation at approximately $2^{\circ}$ eccentricity; in a second task only the fixation spot was visible in an otherwise dark room. Finally, in a third control task the animals initiated a trial by pulling a lever. The monkeys were then presented with a tone and were trained to release the lever immediately after a change in tone pitch. Optical imaging was performed in a window covering the V1 representation of visual field eccentricities ranging from about $1^{\circ}$ to $5^{\circ}$. The time course of the average hemodynamic response over the full area and the neural signal assessed in extracellular recordings at various locations within the imaging field were used to compare electrical and vascular responses.

Upon imaging V1 while the animals performed the fixation-spot-only task, Sirotin and Das observed robust modulations of the hemodynamic signal at the trial frequency, even though the animals were virtually in total darkness and foveal V1, the only region receiving visual input from the fixation point, lay outside of the imaged area. The authors concluded that in some conditions the hemodynamic responses, and thus fMRI signals, too, bear no relation to the underlying regional neural activity, suggesting that the responses reflect "distal neuromodulatory control of cerebral arteries" (Sirotin and Das, 2009). Not surprisingly, the finding sparked a great deal of controversy [e.g. see discussion in references Raichle, 2009; Zheng et al., 2010, and commentary by Kleinschmidt and Müller (2010)], because it challenges the view that fMRI reflects local neural activity. What follows is a discussion of certain claims and conclusions by Sirotin and Das, as well as some comments on the implications of their findings for how to interpret the relationship between fMRI measures and neural signals.

To begin with, it is worth reiterating that fMRI - just like any other methodology, extracellular recordings being no exception - has serious limitations. There is plenty of evidence (see Logothetis, 2008 and references therein) suggesting that the
fMRI signal does not differentiate between function-specific processing and neuromodulation or between bottom-up and top-down signals, and it is likely to confuse excitation and inhibition. In cortical regions in which stimulus- or task-related perceptual or cognitive capacities are instantiated in the activity of a very small number of neurons, volume transmission (Agnati et al., 1992, 2006; Ridet and Privat, 2000; Sykova, 2003) - which probably underlies the altered states of motivation, attention, learning and memory - may affect hemodynamic responses and often make it difficult to deduce the exact role of the area in the task at hand, at least to the extent that this role is thought to be instantiated in the activity of stimulus- or task-selective cortical projection neurons. Even so, the origin of the fMRI signal is at all times neurogenic, and it consistently reflects local changes in overall neural activity.

Interestingly, evidence for the neurogenic basis of the BOLD signal may be found in the same Sirotin-Das (S-D) study that claims to demonstrate a case of neurovascular uncoupling. Specifically, looking at the left spectrogram of Figure 1 (copied from Figure 2 of Sirotin and Das, 2009) one can discern two dark vertical bars (ca. 50\% reduction of the neural signal). The bars are approximately aligned and coextensive with the periods of fixation. The right spectrogram shows the $66-$ to $130-\mathrm{Hz}$ frequency band, and the superimposed white trace depicts the average of power in this range. This trace was vertically stretched at the bottom of Figure 1 to allow visualization of the detailed time course of the neural signal. The blue arrow shows a systematic decrease in LFP, perhaps due to a decrease in spontaneous activity, followed by an overshoot (red arrow) after the fixation period. An inspection of the spectrogram might lead one to expect that even greater signal changes may exist in certain subregions of the frequency domain (e.g. $70-75 \mathrm{~Hz}$ ). The statistical significance of such changes 


\section{Fix-spot and Visual Stimulation}

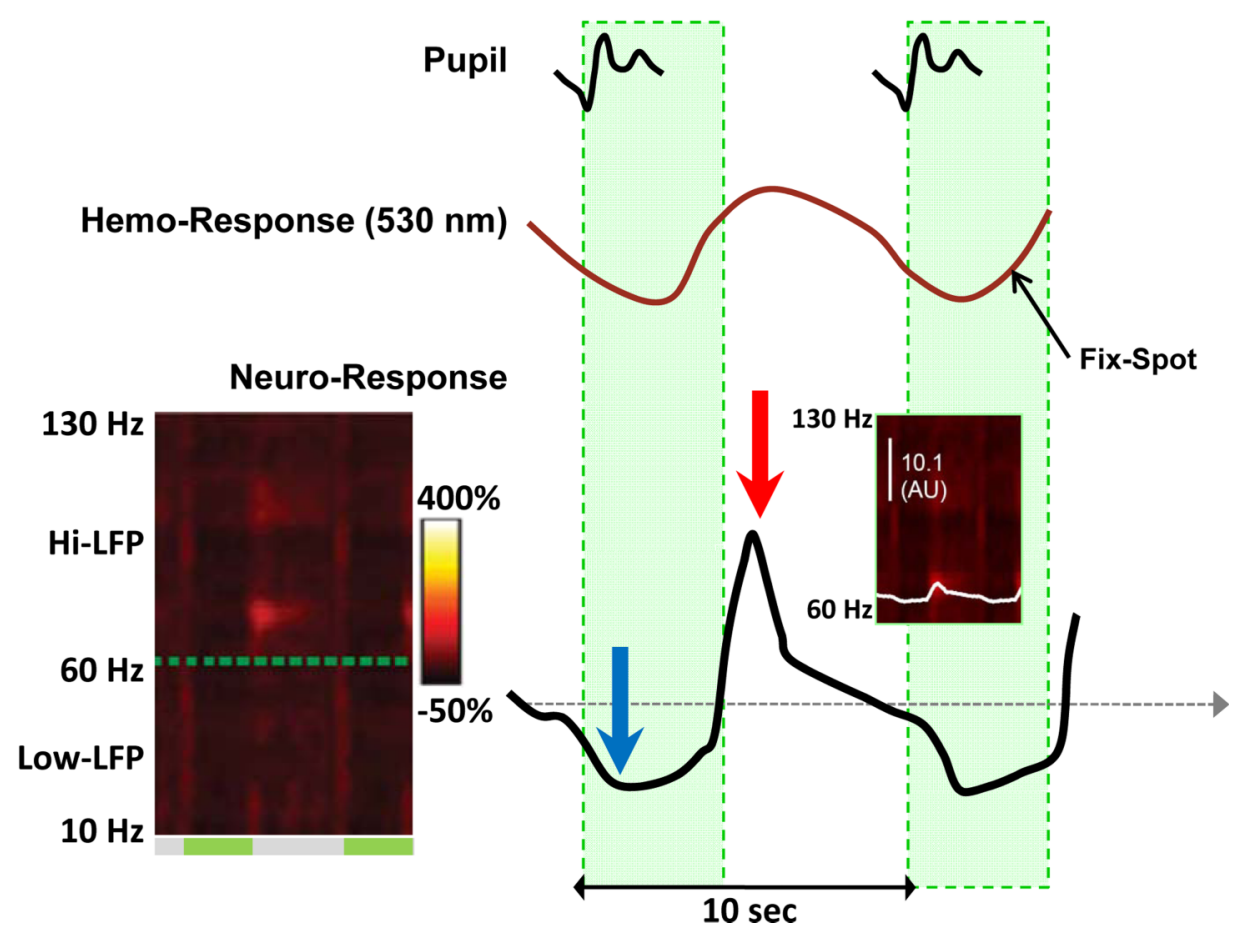

FIGURE 1 | Changes in neural and vascular activity during the performance of the fix-spot-only task. The left spectrogram shows the power of the LFP signal in the $10-$ to $130-\mathrm{Hz}$ range. On the right shown are the trial-triggered papillary (top), hemodynamic (middle), and LFP (bottom) responses (from Figure 2 and Figure S3 in Supplementary Material of the S-D study). An increase in CBV (negative deflection) is evident after the trial onset, followed by an undershoot (positive deflection). The bottom curve is a replica of the white trace shown in the 'Dark' plot of Figure 3A in Supplementary Material of the S-D study (see also inset), but here is vertically stretched to clearly show the activity changes during the task. It represents the trial-triggered power over the hi-LFP frequency range integrated over the $66-$ to $130-\mathrm{Hz}$ range. The blue arrow shows a clear reduction in LFP (evident in the 'dark' band of the spectrogram over all frequencies during the fixation 'green' periods), followed by a rebound (red arrow) activity most prominent in the $70-$ to- $75-\mathrm{Hz}$ band cannot be assessed on the basis of the evidence provided in this study, and they may well be too small to formulate a datasupported hypothesis. Yet a simple qualitative assessment of the figures should be sufficient warning against drawing a conclusion of neurovascular uncoupling, even more so because changes in interneuronal activity might indeed go unnoticed during extracellular recordings.

In multiunit recordings, the electrodes measure changes in the mean extracellular field potential (mEFP) that is mostly due to the distribution of current sources in the dendrites (Avitan et al., 2009). The magnitude of the mEFP in such recordings, as well as that of the signals measured in EEG and MEG, is approximately proportional to the magnitude of the population current dipole $Q$,i.e. the average of each cell's current dipole (Barr et al., 1966; Geselowitz, 1967), and it depends critically on both the strength of individual dipoles and the local geometrical arrangements of neurons (Lorente de No, 1947). Neurons with their dendrites facing in one direction and the somata in the other are said to have an open-field configuration. Experimental and modeling work has shown that open-field generators produce strong individual current dipoles and, under proper geometrical arrangement (e.g. all dendrites along the longitudinal axis, and neurons forming bundles), also powerful population electrical fields that extend over long distances; typical examples are the neocortical and hippocampal pyramidal cells (Murakami et al., 2002, 2003; Murakami and Okada, 2006). On the other hand, cells with radially symmetric dendrites generate electric fields that are confined within the volume of the cells; they are hence considered to be closed-field generators. The small amplitude multiple dipoles, created by action potentials traveling somatofugally in the radially symmetric dendrites tend to diminish, rather than enhance, each other in the extracellular space. Good examples are the aspiny (inhibitory) cortical cells that on average produce $\boldsymbol{Q}$ vectors that are weaker than the supra- and infragranular pyramidal neurons by a factor of up to 8 (Murakami and Okada, 2006). Importantly, the $\boldsymbol{Q}$ of a population of such cells can be much weaker than the linear sum of their individual $\boldsymbol{Q}$ values due to their variable dendritic geometry (Murakami and Okada, 2006). The conclusions drawn from such modeling work are likely to be applicable to the LFPs as well $(0.05$ through $250 \mathrm{~Hz}$ ), since the EEG and LFP signals are correlated to each other (Whittingstall and Logothetis, 2009). It is thus possible in principle that selective activation of certain classes of neurons may have weak effects only on certain LFP bands, and even go unnoticed during extracellular recordings. This is simply a limitation of extracellular recordings that must be taken into account in studies of the neurovascular coupling. Moreover, the fact 
that increased activity of single inhibitory interneurons likely results in vasomotor responses in neighboring brain microvessels (Hamel, 2006) or that astrocytes may respond selectively to sensory stimuli (Schummers et al., 2008) does not by any means imply a lack of regional stimulusor task-related, driver or neuromodulatory cortical neural responses (Chance et al., 2002; McCormick et al., 2003; Hasenstaub et al., 2007).

The responses reported by Sirotin and Das are most likely due to site-specific effects of neuromodulatory input on the cortical excitation-inhibition balance. In their experiments, these authors observed task (i.e. visual or auditory discrimination) independent pupillary responses that systematically preceded the trial-locked hemodynamic fluctuations (Figure 1). In humans such responses are obtained for a wide variety of cognitive processes ranging from simple sensory-event detection through attention, memory, and language processing (Beatty, 1982). They are mediated almost exclusively via norepinephrine (NE) being released from the locus coeruleus (LC). In fact, LC efferents stimulate the iris dilator muscle both directly and indirectly through the Edinger-Westphal nucleus, which in turn projects to the ciliary ganglion (Yoshitomi et al., 1985; Loewenfeld, 1993). Notably, the LC-induced response is distinct from the pupillary light reflex mediated by acetylcholine (Loewenfeld, 1993), so that under constant low-light conditions such as those used in the S-D study, pupil diameter will be a reliable measure of NE-induced neuromodulation (Koss, 1986; Loewenfeld, 1993; Aston-Jones and Cohen, 2005). In other words, during the Sirotin and Das experiments there was a task-induced, modalityindependent periodic activation of the LC of the monkeys.

LC neurons project to most brain regions, including the paleo- and neocortex (Jones et al., 1977), and LC terminals have both nonsynaptic release sites that may provide paracrine-type neurotransmission (Beaudet and Descarries, 1978; Sara, 2009), as well as conventional synapse-like appositions with postsynaptic specializations pointing to the co-existence of wiring transmission (Aston-Jones and Cohen, 2005). NE may increase neural responses by reducing after hyperpolarization (e.g. by blocking $\mathrm{Ca}^{2+}$-dependent $\mathrm{K}^{+}$ currents) or decrease them by enhancing GABA-induced inhibition (Sara, 2009).

In the S-D study, the monkey was involved in a demanding attentional task which required it to continuously discriminate between two point sources with different spectral energy compositions and make a behavioral decision on the basis of the perceived color. During such a task, the release of NE in V1 that follows LC activation is likely to augment the responses induced by the attended fixation spot while decreasing spontaneous activity (the excitation-inhibition balance), as has been shown repeatedly by various investigators, e.g. see review by Aston-Jones and Cohen (Aston-Jones and Cohen, 2005). If reduction in spontaneous neural activity in the non-attended foveal and parafoveal regions imaged in the above study is mediated by increases in interneuronal activity via volume transmission, dissociation between "measurable" neural and vascular signals cannot be excluded (see above), and the increase in CBV observed by the authors in the "hitherto unknown signal" (Figure 1, middle red trace) may simply reflect increased metabolism due to increases in inhibitory conductance.

An interesting observation in the S-D study is the specificity of V1 neuromodulation. The LC-NE system was initially viewed as having relatively broad and spatially nonspecific effects on cortical information processing. But later studies showed that LC projections, although widespread, exhibit substantial regional and laminar specificity. In monkeys, for instance, the laminar pattern of noradrenergic innervation in primary visual cortex differs fundamentally from that in both prefrontal and primary somatosensory cortices (Morrison et al., 1982; Foote et al., 1983). A large number of studies have demonstrated that the effects of the LC-NE system on target neurons are characterized by temporal and spatial discreteness. In other words, the LC-NE system acts on a discrete, specifiable set of neurons under a particular set of circumstances to produce a defined set of effects (Foote et al., 1983). The regional anatomical-functional specificity is also congruent with the observation that bursts of LC activity are correlated with the outcome of task-related decision processes (see references in the review by Aston-Jones and Cohen, 2005). Interestingly, the S-D study also showed that LC responses, evident via the pupillary response, induce a task- and modality specific change in hemodynamics: no CBV changes in V1 were measured during the auditory control task. By combining broad access and specificity, the massive efferent system of the LC is likely to impose simultaneous internal-state changes on many different brain regions subject to sensory stimulation.

In summary, Sirotin and Das addressed an important question using an outstanding methodology. In agreement with previous studies (see above), they demonstrated that their task selectively modulates neuronal activity in $\mathrm{V} 1$, likely reducing spontaneous activity (e.g. for gain control, Chance et al., 2002, but see also alternative observations, McCormick et al., 2003; Hasenstaub et al., 2007). The increased inhibition, visible in their spectrograms, may trigger CBV changes and yield the "anticipatory responses". Yet, the authors' generalizations are unjustified, and I suspect that their conclusions are incorrect.

The fMRI methodology is used extensively in cognitive neuroscience, and as in any advanced field of investigation there are quite a few publications that are senseless and do not enhance our understanding of brain function. The problem, however, is not in the methodology but rather in those investigators who use it without understanding its limitations. Even if neurovascular coupling were well understood, the limitations of fMRI reflecting mass action (Logothetis, 2008) would still require a multimodal approach before we can even begin to fathom the exact neural mechanisms underlying the usual fMRI activations and understand the role of the mapped areas. This having been said, and despite its shortcoming, fMRI is a useful and meaningful tool for the study of brain function, and fMRI signals reflect changes in regional neuronal mass activity.

\section{ACKNOWLEDGMENTS}

I thank my colleagues Christoph Kayser, Oxana Eschenko, Ahalya Viswanathan, and Brian Wandell for insightful comments on the final version of the article.

\section{REFERENCES}

Agnati, L. F., Bjelke, B., and Fuxe, K. (1992). Volume transmission in the brain. Am. Sci. 80, 362-373.

Agnati, L. F., Leo, G., Zanardi, A., Genedani, S., Rivera, A., Fuxe, K., and Guidolin, D. (2006). Volume 
transmission and wiring transmission from cellular to molecular networks: history and perspectives. Acta Psychol. (Amst) 187, 329-344.

Aston-Jones, G., and Cohen, J. D. (2005). An integrative theory of locus coeruleus-norepinephrine function: adaptive gain and optimal performance. Annu. Rev. Neurosci. 28, 403-450.

Avitan, L., Teicher, M., and Abeles, M. (2009). EEG generator-a model of potentials in a volume conductor. J. Neurophysiol. 102, 3046-3059.

Barr, R. C., Pilkington, T. C., Boineau, J. P., and Spach, M. S. (1966). Determining surface potentials from current dipoles with application to electrocardiography. IEEE Trans. Biomed. Eng. 13, 88-92.

Beatty, J. (1982). Task-evoked pupillary responses, processing load, and the structure of processing resources. Psychol. Bull. 91, 276-292.

Beaudet, A., and Descarries, L. (1978). Mono-amine innervation of rat cerebral-cortex - synaptic and non-synaptic axon terminals. Neuroscience 3, 851-860.

Bonhoeffer, T., and Grinvald, A. (1996). "Optical imging based on intrinsic signals," in Brain Mapping, The Methods, eds A. W. Toga and J. C. Mazziotta (New York: Academic Press), 55-97.

Chance, F. S., Abbott, L. F., and Reyes, A. D. (2002). Gain modulation from background synaptic input. Neuron 35, 773-782.

Dale, A. M., and Halgren, E. (2001). Spatiotemporal mapping of brain activity by integration of multiple imaging modalities. Curr. Opin. Neurobiol. 11, 202-208.

Foote, S. L., Bloom, F. E., and Astonjones, G. (1983). Nucleus locus ceruleus - new evidence of anatomical and physiological specificity. Physiol. Rev. 63, 844-914.

Geselowitz, D. B. (1967). On bioelectric potentials in an inhomogeneous volume conductor. Biophys. J. 7, 1-11.

Goense, J. B., and Logothetis, N. K. (2008). Neurophysiology of the BOLD fMRI signal in awake monkeys. Curr. Biol. 18, 631-640.

Hamel, E. (2006). Perivascular nerves and the regulation of cerebrovascular tone. J. Appl. Physiol. 100 1059-1064.

Hasenstaub, A., Sachdev, R. N. S., and McCormick, D. A. (2007). State changes rapidly modulate cortical neuronal responsiveness. J. Neurosci. 27, 9607-9622.
Jones, B. E., Halaris, A. E., Mcilhany, M., and Moore, R. Y. (1977). Ascending projections of locus coeruleus in rat.1. Axonal-transport in central noradrenaline neurons. Brain Res. 127, 1-21.

Kleinschmidt, A., and Müller, N. G. (2010). The blind, the lame, and the poor signals of brain function-a comment on Sirotin and Das (2009). Neuroimage 50, 622-625.

Koss, M. C. (1986). Pupillary dilation as an index of central nervous system alpha 2-adrenoceptor activation. J. Pharmacol. Methods 15, 1-19.

Loewenfeld, I. (1993). The Pupil: Anatomy, Physiology, and Clinical Applications. Detroit: Wayne State University Press.

Logothetis, N. K. (2008). What we can do and what we cannot do with fMRI. Nature 453, 869-878.

Logothetis, N. K., Pauls, J., Augath, M., Trinath, T., and Oeltermann, A. (2001). Neurophysiological investigation of the basis of the fMRI signal. Nature 412 , 150-157.

Logothetis, N. K., and Wandell, B. A. (2004). Interpreting the BOLD signal. Annu. Rev. Physiol. 66, 735-769.

Lorente de No, R. (1947). Analysis of the distribution of action currents of nerve in volume conductors. In studies from the Rockefeller Institute Medical Research. A Study of Nerve Physiology 132, 384-477.

McCormick, D. A., Shu, Y. S., and Hasenstaub, A. (2003) "Balanced recurrent excitation and inhibition in local cortical networks," in Excitatory-Inhibitory Balance: Synapses, Circuits, and Systems Plasticity, ed. T. Hensch (New York: Kluwer Academic Press).

Morrison, J. H., Foote, S. L., Oconnor, D., and Bloom, F. E. (1982). Laminar, tangential and regional organization of the noradrenergic innervation of monkey cortex-dopamine-beta-hydroxylase immunohistochemistry. Brain Res. Bull. 9, 309-319.

Murakami, S., Hirose, A., and Okada, Y. C. (2003). Contribution of ionic currents to magnetoencephalography (MEG) and electroencephalography (EEG) signals generated by guinea-pig CA3 slices. J. Physiol. (Lond.) 553, 975-985.

Murakami, S., and Okada, Y. (2006). Contributions of principal neocortical neurons to magnetoencephalography and electroencephalography signals. J. Physiol. (Lond.) 575, 925-936.

Murakami, S., Zhang, T. S., Hirose, A., and Okada, Y. C. (2002). Physiological origins of evoked magnetic fields and extracellular field potentials produced by guinea-pig CA3 hippocampal slices. J. Physiol. (Lond.) 544, 237-251.

Raichle, M.E. (2009). A paradigm shift in functional brain imaging. J. Neurosci. 29, 12729-12734.

Rauch, A., Rainer, G., and Logothetis, N. K. (2008). The effect of a serotonin-induced dissociation between spiking and perisynaptic activity on BOLD functional MRI. Proc. Natl. Acad. Sci. U.S.A. 105, 6759-6764.

Ridet, J. L., and Privat, A. (2000). Volume transmission. Trends Neurosci. 23, 58-59.

Sara, S. J. (2009). The locus coeruleus and noradrenergic modulation of cognition. Nat. Rev. Neurosci. 10, 211-223.

Schummers, J., Yu, H. B., and Sur, M. (2008). Tuned responses of astrocytes and their influence on hemodynamic signals in the visual cortex. Science 320, 1638-1643.

Sirotin, Y. B., and Das, A. (2009). Anticipatory haemodynamic signals in sensory cortex not predicted by local neuronal activity. Nature 457, 475-479.

Sykova, E. (2003). Volume transmission in health and disease: communication via the brain extracellula space. J. Neurochem. $85,2$.

Whittingstall, K., and Logothetis, N. K. (2009). Frequencyband coupling in surface EEG reflects spiking activity in monkey visual cortex. Neuron 64, 281-289.

Yoshitomi, T., Ito, Y., and Inomata, H. (1985). Adrenergic excitatory and cholinergic inhibitory innervations in the human iris dilator. Exp. Eye Res. 40, 453-459.

Zheng, Y., Pan, Y., Harris, S., Billings, S., Coca, D., Berwick, J., Jones, M., Kennerley, A., Johnston, D., Martin, C., Devonshire, I. M., and Mayhew, J. (2010). A dynamic model of neurovascular coupling: implications for blood vessel dilation and constriction. [Epub ahead of print].

Received: 26March 2010; accepted:04 April 2010; published online: 02 June 2010.

Citation: Logothetis NK (2010) Neurovascular uncoupling: much ado about nothing. Front. Neuroenerg. 2:2. doi: 10.3389/fnene.2010.00002

Copyright $($ C 2010 Logothetis. This is an open-access article subject to an exclusive license agreement between the authors and the Frontiers Research Foundation, which permits unrestricted use, distribution, and reproduction in any medium, provided the original authors and source are credited. 